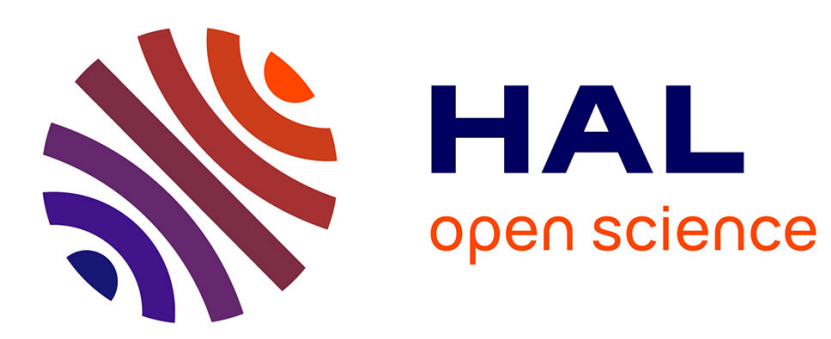

\title{
Concurrent Undo Operations in Collaborative Environments using Operational Transformation
}

\author{
Jean Ferrié, Nicolas Vidot, Michelle Cart
}

\section{To cite this version:}

Jean Ferrié, Nicolas Vidot, Michelle Cart. Concurrent Undo Operations in Collaborative Environments using Operational Transformation. CoopIS: Cooperative Informations Systems, Oct 2004, Agia Napa, Cyprus. pp.155-173, 10.1007/978-3-540-30468-5_12 . lirmm-00108870

\section{HAL Id: lirmm-00108870 \\ https://hal-lirmm.ccsd.cnrs.fr/lirmm-00108870}

Submitted on 23 Sep 2019

HAL is a multi-disciplinary open access archive for the deposit and dissemination of scientific research documents, whether they are published or not. The documents may come from teaching and research institutions in France or abroad, or from public or private research centers.
L'archive ouverte pluridisciplinaire HAL, est destinée au dépôt et à la diffusion de documents scientifiques de niveau recherche, publiés ou non, émanant des établissements d'enseignement et de recherche français ou étrangers, des laboratoires publics ou privés. 


\title{
Concurrent Undo Operations in Collaborative Environments Using Operational Transformation
}

\author{
Jean Ferrié ${ }^{1}$, Nicolas Vidot ${ }^{2}$, and Michelle Cart ${ }^{1}$ \\ ${ }^{1}$ LIRMM, Montpellier II University \\ 161, rue Ada, 34392 Montpellier (France) \\ \{ferrie, cart\}@lirmm. fr \\ ${ }^{2}$ GRIMAAG, Université des Antilles et de la Guyane \\ B.P. 7209, 97275 Schœlcher Cedex, Martinique (France) \\ http://www.martinique.univ-ag.fr
}

\begin{abstract}
In distributed collaborative systems, replicated objects, shared by users, are subject to concurrency constraints. All methods [4, 13, 18, 15, 16, 19, 22] proposed to serialize concurrent operations and achieve copies convergence of replicated objects are based on the use of Operational Transformations. In this context, giving the user the ability to undo an operation has been recognized as a difficult problem $[1,2,3,12,14,20,21]$. The few general propositions to solve the problem sometimes compromise copies convergence and/or users' intention, insofar as the Operational Transformations used are unsuitable for undo. This paper has a twofold objective. Firstly, it aims to highlight two general conditions (named $\mathrm{C} 3$ and $\mathrm{C} 4$ ) that need to be satisfied by any transformation adapted to undo. Secondly, it presents a general undo algorithm based on the definition of a generic undo-fitted transformation, which automatically verifies these conditions. The interest of the proposed method is that the undoing of an operation obeys to the same processing as the one used for regular operations in collaborative systems such as $[15,19]$.
\end{abstract}

Keywords: Distributed collaborative systems, copies consistency, operational transformations, concurrent undo

\section{Introduction}

The purpose of a collaborative system is to facilitate team working and, in particular, to enable the manipulation of shared objects by members of a team whilst making them evolve in a coherent way. Usually, a shared object involved in a collaborative activity (shared text edition, shared CAD, electronic conferences, etc.) is subject to concurrent accesses and real-time constraints. The real-time aspect necessitates every user seeing the effects of his own actions on the object immediately, and the effects resulting from the actions of other users as soon as possible. In a distributed system when assuming non-negligible network latency, this high reactivity cannot be achieved unless each object is replicated on every site. Consequently, the problem is to conciliate both real-time constraint and consistency preservation of object copies, as they can be modified concurrently by many users. 
In this context, various algorithms [4, 13, 18, 15, 16, 19, 22], which exploit the semantic properties of the operations on the objects, have been proposed to serialize concurrent operations and thus ensure the convergence of all copies of an object. All these algorithms, which are based on Operational Transformations, exploit a transposition function to transform an operation before integrating it into the history associated with an object copy so as to respect user intention in case of concurrency. The same problem is found in configuration management [9]. In these contexts, giving the user the ability to undo an operation has been recognized as a difficult problem $[1,2,3,12,14,20,21]$, when taking concurrency between operations into account. In [20] an undo algorithm ANYUNDO was proposed to enable a user to undo any operation (local or remote) that has been executed on the object. The action of undoing an operation is based on the generation of the inverse operation and the transformation of the latter to take concurrent operations into account. Unfortunately, some critical situations can compromise the convergence of the copies and/or the user intention. In a recent paper [21], corrections to ANYUNDO algorithm are made in order to remedy some critical situations. Such situations are avoided in adOPTed [14], at the expense of a restrictive undo policy which only allows local operations to be undone in the reverse execution order. The lack of generality of these algorithms is due to the fact that the Operational Transformations used are not well suited to undo. To obtain a correct result, the transposition function would have to satisfy two conditions (called C3 and C4) highlighted by our study. These conditions are difficult to check in practice. In this context, our approach proposes a general undo algorithm which automatically satisfies conditions C3 and C4 thanks to the definition of a generic Operational Transformation adapted to undo.

The paper is organized as follows. Section 2 describes the model used along with the use of the Operational Transformations to ensure the consistency of the copies of an object in distributed collaborative environments. Section 3 describes the problems presented by the undo of an operation and the conditions that must be met by the inverse operation to ensure that the action of undoing an operation is carried out correctly. Section 4 details the principles of the general undo algorithm. Section 5 illustrates how it works with an example. Section 6 compares it with the other known algorithms.

\section{Operational Transformations}

A distributed collaborative system is constituted from a set of sites interconnected by a supposed reliable network. Each object shared by the users is replicated so that a copy of the object exists on every site and it can be handled using definite operations. In order to maintain consistency between copies, every operation generated and executed on a site must be executed on all other copies as well. This requires every operation generated on a site to be broadcast to the other sites; after reception on a site, the operation is executed on the local copy of the object. Given a site, a local operation is an operation generated on this site whereas a remote operation is one that has been generated on another site. In order to guarantee users a minimum response time, operations generated on a site (i.e. local operations) are executed immediately on this site. 
This section reviews the three constraints encountered when trying to achieve consistency maintenance of object copies and outlines the principles of their solutions: (1) causality preservation, (2) user intention preservation and (3) convergence. A collaborative text editor will be used as an example. Let us assume a text is an ordered collection of sentences, each one being an object represented by a string of characters. The operations defined on this object are:

insert $(p, c):$ inserts character $c$ at position $p$ in the string,

$\operatorname{delete}(\boldsymbol{p})$ : deletes character at position $p$ in the string.

In the following, we suppose that users are working concurrently and are modifying the same sentence.

\subsection{Causality Preservation}

An operation op $\mathrm{p}_{1}$ is said to causally precede op (noted op $_{1}$ precedes $_{C} \mathrm{op}_{2}$ ) iff $\mathrm{op}_{2}$ was generated on a site after $\mathrm{op}_{1}$ has been executed on this site. Consequently, $\mathrm{op}_{2}$ is supposed to depend on the effects of operation $\mathrm{op}_{1}$. Causality preservation ensures that all operations related by a causality relation are executed in the same order on every copy. It is achieved in the majority of the methods [4, 13, 18, 15, 19], by using a state vector associated with each site and each object and by timestamping each operation. Instead of state vectors, method [22] uses continuous timestamps delivered by a sequencer which, when associated with a differed broadcast, makes it possible to ensure a sequential reception compatible with the causal reception.

\subsection{User Intention Preservation}

Operations that are not causally related are said to be concurrent. In other words op and $\mathrm{op}_{2}$ are concurrent iff neither (op precedes $\left._{\mathrm{C}} \mathrm{op}_{2}\right)$ nor $\left(\mathrm{op}_{2}\right.$ precedes $\left._{\mathrm{C}} \mathrm{op}_{1}\right)$. In this case, neither one depends on the effects of the other. Thus, they can be executed in any order on the different sites. Nevertheless, if a site executes op $\mathrm{p}_{1}$ before $\mathrm{op}_{2}$, it must take into account the changes made by $\mathrm{op}_{1}$ when it executes $\mathrm{op}_{2}$ so as the intention of the user who generated $\mathrm{op}_{2}$ to be respected. The intention of a user may be for instance to add 's' at the end of a word or to double a letter in a word. This intention is achieved by the execution of an operation which is relative to a specific state of the object. In the example of Figure 1-a, two users work simultaneously on the same object whose state is "efect". The intention of user 1 is to add ' $f$ ' to obtain "effect". This is achieved by operation insert $(2$, ' $f$ '). The intention of user 2 is to add 's' at the end of the word which is achieved by the operation insert $(6$, 's'). When this operation is delivered and executed on site 1, the new state is "effecst" which is not what user 2 expected. To respect his intention, operation insert $(6$, 's') needs to be transformed on site 1 in order to execute insert(7, 's') instead of insert(6, 's') (see Figure 1-b).

User intention preservation ensures that the execution of an operation op on each copy has an effect that achieves the intention of the user at the time when op was generated. The problem of user intention preservation is due to the fact that an operation generated on a site achieves user intention depending on the state of the copy on this site. If this operation were to be executed on a remote site after the execution of a concurrent operation, it might no longer achieve the initial intention in 
the case of the state of the copy not being the same. The solution to this problem is based on the use of Operational Transformations. This consists of transforming every remote operation to be executed so that it takes into account the modifications made by all the concurrent operations serialized before it. This transformation is possible provided that a function specific to the semantics of the operations is defined which gives for all pairs of operations $\left(\mathrm{op}_{1}, \mathrm{op}_{2}\right)$ an operation written as $\mathrm{op}_{2}{ }^{\mathrm{op}}{ }_{1}$, which is defined for the state resulting from the execution of $\mathrm{op}_{1}$ and which achieves the same intention as $\mathrm{op}_{2}$. This transformation function introduced in [4] is also used in other systems [13, 18, 16, 19, 22] under various denominations. We call it forward transposition.

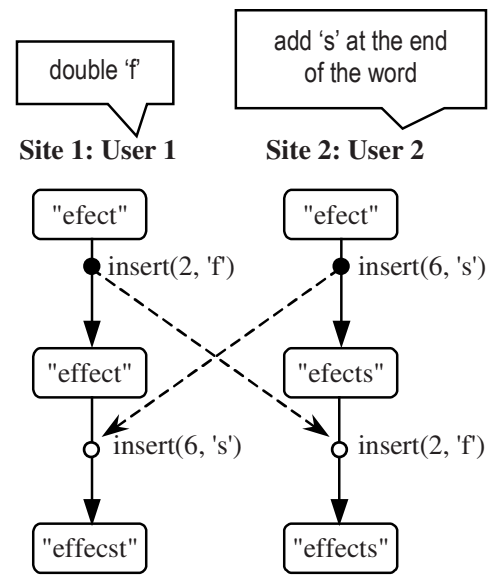

a) Non-respect of user 2 intention, on site 1

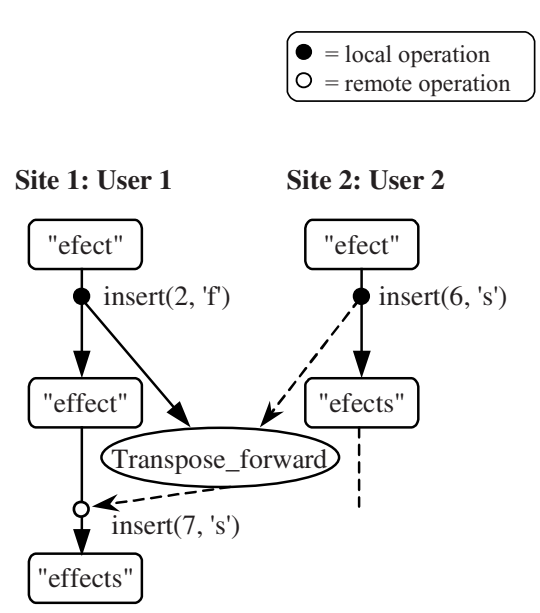

b) Use of the forward transposition to ensure the respect of user 2 intention, on site 1

Fig. 1. Respecting the intention of the user

Let $\mathrm{O}_{\mathrm{i}}$ be the initial state of the object, $\mathrm{O}_{i}$.op the state obtained after the execution of op and Intention(op, $\mathrm{O}_{\mathrm{i}}$ ), the intention which is achieved by operation op on object state $\mathrm{O}_{\mathrm{i}}$. The forward transposition is then formally defined as follows:

$$
\begin{gathered}
\text { Transpose_forward }\left(\mathrm{op}_{1}, \mathrm{op}_{2}\right)=\mathrm{op}_{2}{ }^{\mathrm{op}}{ }_{1} \\
\text { with: } \forall \mathrm{O}_{\mathrm{i}} \text {, Intention }\left(\mathrm{op}_{2}{ }^{\mathrm{op}}{ }_{1}, \mathrm{O}_{\mathrm{i}} . \mathrm{op}_{1}\right)=\operatorname{Intention}\left(\mathrm{op}_{2}, \mathrm{O}_{\mathrm{i}}\right) \text {. }
\end{gathered}
$$

Figure 1-b depicts the effect of the forward transposition for the pair of operations (insert(2, 'f'), insert(6, 's')). More generally, let seqn be a sequence of $n$ operations; the forward transposition of operation op with $\operatorname{seq}_{n}$, noted $\mathrm{op}^{\mathrm{seq}_{\mathrm{n}}}$, is defined recursively by: $\mathrm{op}^{\mathrm{seq}_{\mathrm{n}}}=$ Transpose_forward $\left(\mathrm{op}_{\mathrm{n}}, \mathrm{op}_{\mathrm{n}-1}^{\mathrm{seq}}\right)$ with $\mathrm{seq}_{\mathrm{n}}=\mathrm{op}_{1} \cdot \mathrm{op}_{2} \ldots . \mathrm{op}_{\mathrm{n}}=\mathrm{seq}_{\mathrm{n}-1} \cdot \mathrm{op}_{\mathrm{n}}$ and $\mathrm{op}^{\text {seq }}{ }_{0}=\mathrm{op}$, where op $\mathrm{p}_{\mathrm{i}} . \mathrm{p}_{\mathrm{j}}$ represents the execution of op $\mathrm{p}_{\mathrm{i}}$ followed by the execution of opj.

It is important to note that the forward transposition requires both operations to be defined with the object in the same state. To satisfy this requirement in all situations, 
different solutions have been proposed in order to apply forward transposition in the right way.

In [18] operation op 1 is transformed using the reverse function of forward transposition (called Exclusion_Transformation), so that it is defined for the same state as $\mathrm{op}_{2}$ and enables the use of the forward transposition. In [13] several equivalent histories which respect the causal order are kept on each site so that the intermediate states of the object can be retrieved on each site. In $[11,15,19]$ a new transformation is defined. This function [11] which we call backward transposition makes it possible to change the execution order of a pair of operations while respecting user intention. More accurately, the backward transposition of a couple of operations $\left(\mathrm{op}_{1}, \mathrm{op}_{2}\right)$, executed in this order, gives as a result the couple $\left(\mathrm{op}_{2}{ }^{\prime}, \mathrm{op}_{1}{ }^{\prime}\right)$ corresponding to their execution in reverse order which leads to the same state, and is compatible with the forward transposition. Formally:

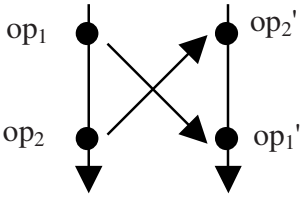

$\mathrm{op}_{1} . \mathrm{op}_{2} \equiv \mathrm{op}_{2}{ }^{\prime} . \mathrm{op}_{1}{ }^{\prime}$

$$
\begin{array}{r}
\text { Transpose_backward }\left(\mathrm{op}_{1}, \mathrm{op}_{2}\right)=\left(\mathrm{op}_{2}{ }^{\prime}, \mathrm{op}_{1}{ }^{\prime}\right) \\
\text { with: } \mathrm{op}_{2}=\text { Transpose_forward }\left(\mathrm{op}_{1}, \mathrm{op}_{2}{ }^{\prime}\right) \text { and } \\
\mathrm{op}_{1}{ }^{\prime}=\text { Transpose_forward }\left(\mathrm{op}_{2}{ }^{\prime}, \mathrm{op}_{1}\right)
\end{array}
$$

The backward transposition is only defined for a sequence of operations $\left(\mathrm{op}_{1}, \mathrm{op}_{2}\right)$ obtained from concurrent operations $\left(\mathrm{op}_{1}, \mathrm{op}_{2}{ }^{\prime}\right)$. Both forward and backward transpositions are examples of what is called Operational Transformation. In the following, these Operational Transformations are applied to objects of the type "string of characters". They are applied to XML objects in [9], and spreadsheets objects in [10]. They are also applied recursively over the different levels of a tree representation of documents in [6].

\subsection{Copies Convergence}

Taking into account causality as well as user intention is not always sufficient to achieve executions that guarantee the convergence of the copies on all sites. Indeed, as concurrent operations can be executed in any order on different sites, the forward transposition needs to verify two conditions $[4,13]$. The first condition $\mathrm{C} 1$, ensures that, starting from the same state, the execution of $\mathrm{op}_{1}$ followed by the execution of $\mathrm{op}_{2}{ }^{\mathrm{op}}{ }_{1}$ produces the same state as the execution of $\mathrm{op}_{2}$ followed by the execution of $\mathrm{op}_{1}{ }^{\mathrm{op}}{ }_{2}$. It is formally defined as:

Condition C1. Let op $\mathrm{p}_{1}$ and $\mathrm{op}_{2}$ be two concurrent operations defined on the same state. The forward transposition verifies $\mathrm{C} 1$ iff:

$$
\mathrm{O}_{1} \cdot \mathrm{op}_{1} \cdot \mathrm{op}_{2}{ }^{\mathrm{op}}{ }_{1} \equiv \mathrm{O}_{\mathrm{i}} \cdot \mathrm{op}_{2} \cdot \mathrm{op}_{1}{ }^{\mathrm{op}}{ }_{2}
$$

where $\equiv$ denotes the equivalence of states obtained after applying both sequences from the same state $\mathrm{O}_{i}$. 
Figure 2 gives an example of a forward transposition verifying condition $\mathrm{C} 1$. In the case of concurrent insertions of different characters at the same position $\left(\mathrm{p}_{1}=\mathrm{p}_{2}\right)$, the alphabetical order (noted $\operatorname{pr}())$ is arbitrarily privileged. In the case of concurrent insertions of the same character, only one character is inserted, and the returned operation is identity (id).

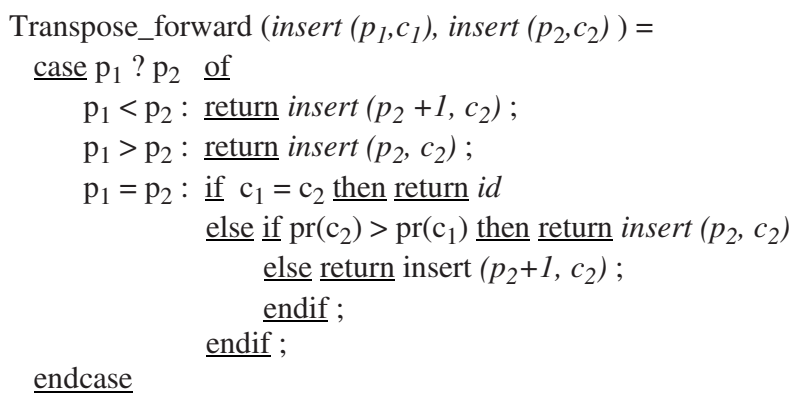

Fig. 2. Example of a forward transposition verifying $\mathrm{C} 1$

The second condition C2, ensures that the forward transposition of an operation with a sequence of two or more concurrent operations does not depend on the order used to serialize these operations. It is formally defined as follows:

Condition C2. Whatever operations $\mathrm{op}_{1}, \mathrm{op}_{2}$ and $\mathrm{op}_{3}$ are, the forward transposition verifies C2 iff:

$$
\mathrm{op}_{3}{ }^{\mathrm{op} 1: \mathrm{op}_{2}}=\mathrm{op}_{3}{ }^{\mathrm{op_{2 } : \mathrm { op } _ { 1 }}}
$$

where the notation op $\mathrm{p}_{\mathrm{i}}: \mathrm{op}_{\mathrm{j}}$ denotes $\mathrm{op}_{\mathrm{i}} \cdot \mathrm{op}_{\mathrm{j}}{ }_{\mathrm{i}}{ }_{\mathrm{i}}$.

Most methods, adOPTed [13], SOCT2 [15, 16] and GOTO [19] are based on satisfying conditions $\mathrm{C} 1$ and $\mathrm{C} 2$. In [18] conditions $\mathrm{C} 1$ and $\mathrm{C} 2$ are not required but a unique serialization order which complies with the causal order is imposed for the operations on all the sites; unfortunately, it may be necessary to Undo/Redo some operations to conform to this order. In [4], condition C2 is not required to the detriment of the convergence of the copies. In [22], condition C2 is not needed thanks to the implementation of a unique and continuous serialization order, given by a sequencer.

\subsection{Principles of Collaborative Algorithms}

Generally speaking, the principle of collaborative algorithms capable of ensuring the consistency of the copies involves memorizing the history of the operations executed from the initial state to the current state for each copy of object. Any operation generated locally is executed immediately before being added to the history. The reception of a remote operation $\mathrm{OP}$ requires a phase of integration to determine the operation $\mathrm{OP}^{\prime}$ achieving the same intention as $\mathrm{OP}$, to be executed on the current state. The difference between the algorithms lies in how they transform the received operation OP. For instance, in the algorithms such as SOCT2 [15, 16] or GOTO [19], when a site receives a remote operation OP, it determines the sequence seqconc of concurrent operations, then executes the processing shown in Figure 3. 
1. Forward transpose OP with the sequence of concurrent operations, noted seqconc, to obtain operation OP' such that:

Transpose_forward (seqconc, $\mathrm{OP})=\mathrm{OP}^{\prime}$

2. Execute $\mathrm{OP}^{\prime}$ on the current state

3. Append OP' to the local history

Fig. 3. Processing of a remote operation once received by a site

In the following, we show how to undo any operation (do or undo) so that the undo operation is processed in the same way as any other operation OP, while respecting the three constraints: (1) causality preservation, (2) user intention preservation and (3) copies convergence.

\section{Undo Problems}

Let op be the operation to achieve intention $\mathrm{I}$, executed on the object $\mathrm{O}$ from the initial state $\mathrm{O}_{\mathrm{i}}$. Let us consider the sequence seq of (n-1) operations (with seq = $\left.\mathrm{op}_{1} . \mathrm{op}_{2} \ldots . \mathrm{op}_{\mathrm{n}-1}\right)$, respectively, to achieve the intentions $\mathrm{I}_{1}, \mathrm{I}_{2}, \ldots \mathrm{I}_{\mathrm{n}-1}$ executed in this order starting from the state $\mathrm{O}_{i}$.op.

Undoing operation op consists of generating and executing, at the current state $\mathrm{O}_{\mathrm{i}}$.op.seq, the operation $\mathrm{op}_{\mathrm{n}+1}$ that cancels the effects of op without modifying the intentions of the other operations. This operation must lead the object to the same state as the sequence seq' of the (n-1) operations (with seq' $=o_{1}{ }^{\prime} . \mathrm{op}_{2}{ }^{\prime} \ldots . . \mathrm{op}_{\mathrm{n}-1}{ }^{\prime}$ ), where these operations achieve the same intentions $\mathrm{I}_{1}, \mathrm{I}_{2}, \ldots \mathrm{I}_{\mathrm{n}-1}$ and are executed in this order starting from the initial state $\mathrm{O}_{\mathrm{i}}$. In other words: $\mathrm{O}_{\mathrm{i}}$.op.seq.op $\mathrm{p}_{\mathrm{n}+1}=\mathrm{O}_{\mathrm{i}}$. seq'.

The operation op $\mathrm{p}_{\mathrm{n}+1}$ can be obtained using two different strategies.

Strategy 1. It consists in generating operation $\overline{\mathrm{op}}$, the inverse operation of op, from state $\mathrm{O}_{\mathrm{i}}$.op and considering it as an operation concurrent with the sequence of operations seq. Thus $\overline{\mathrm{op}}$ must be forward transposed with seq; the operation obtained $\overline{\mathrm{op}} \mathrm{seq}$ can then be executed on the current state. This strategy and the algorithm called naïve algorithm of undo which implements it are illustrated by Figure 4 . The algorithm is executed on the site where a decision is made to undo; the operation op ${ }^{s e q}$, which is broadcast to the other sites, is processed on these sites like a regular operation.

Strategy 2. It consists in backward transposing the pair (op, seq) so as to obtain an equivalent history (seq', op') in which the operation op' (i.e. opseq') to be undone is the last one executed. To undo op, it then suffices to generate operation $\overline{\mathrm{op}}$ ', the inverse operation of op', and to execute it on the current state. This strategy and the algorithm which implements it are illustrated by Figure 5. 


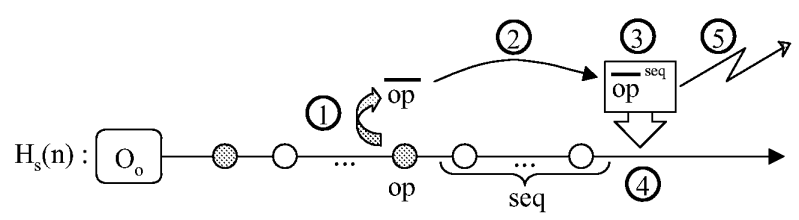

1. Generate the inverse of op, noted $\overline{\mathrm{op}}$, on the state $\mathrm{O}_{\mathrm{i}}$.op such that: $\mathrm{O}_{\mathrm{i}} \cdot \mathrm{op} . \overline{\mathrm{op}}=\mathrm{O}_{\mathrm{i}}$

2. Calculate $\overline{\mathrm{op}} \mathrm{seq}=$ Transpose forward (seq, $\overline{\mathrm{op}})$

3. Execute $\overline{\mathrm{op}}$ seq on the current state $\mathrm{O}_{\mathrm{i}}$.op.seq

4. Append $\overline{o p}$ seq to local history

5. Broadcast $\overline{\mathrm{op}} \mathrm{seq}$ to other sites

Fig. 4. Undoing op according to Strategy 1

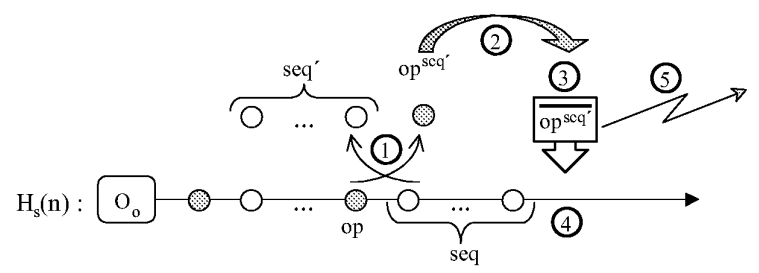

\footnotetext{
1. Calculate (seq', op') = Transpose_backward (op, seq)

with op' = opseq'

2. Generate the inverse of opseq', i.e. $\overline{\text { opseq }}$

3. Execute $\overline{\mathrm{op}^{\mathrm{seq}}}$ on the current state $\mathrm{O}_{\mathrm{i}}$.op.seq

4. Append opseq to the local history

5. Broadcast opseq to other sites
}

Fig. 5. Undoing op according to Strategy 2

Strategy 2 was first proposed and used in [12]. All other existing systems in which it is possible to undo and which are based on the Operational Transformations [14, 20, 21] use Strategy 1. They consider the undoing of an operation op as the generation of the inverse operation $\overline{\mathrm{o}}$ Insofar as the inverse operation is regarded as a regular operation, this process ignores the specificity of undo and fails to observe the conditions needed to ensure the correction of the undo algorithm.

\subsection{Neutrality of the Do/Undo Pair for the Transposition (Condition C3)}

To ensure the preservation of user intention when undoing operation, constraints on the forward transposition must be satisfied. This is illustrated by the example in Figure 6. To undo $\mathrm{op}_{1}$ on site 1, the naïve algorithm based on Strategy 1 leads to generation and execution of $\mathrm{op}_{2}=\bar{\sigma}_{1}$, which is then broadcast to site 2 . When operation $\mathrm{op}_{3}=\operatorname{insert}(2$, 'a'), which carries out the intention to insert 'a' after 'b', is received on site 1 it is regarded as being concurrent with $\mathrm{op}_{1}$ and $\mathrm{op}_{2}$. As a result, it is forward transposed successively with $\mathrm{op}_{1}$ and $\mathrm{op}_{2}$ to give the operation $\mathrm{op}_{3} \mathrm{op}_{1}$. $\mathrm{op}_{2}=$ in$\operatorname{sert}(1$, 'a') whose execution leads to the state "ab". In this example, the copies 
converge towards the same state "ab" and the undoing of $\mathrm{op}_{1}$ strictly respects the intention of user 1 since 'b' was not removed. However, the intention of user 2 was not respected insofar as 'a' was inserted before ' $b$ '. In fact, the transposition of $\mathrm{op}_{3}$ with the sequence seq $=\mathrm{op}_{1} \cdot \mathrm{op}_{2}=\mathrm{op}_{1} \cdot \mathrm{op}_{1}$ should have resulted in $\mathrm{op}_{3}$. In other words, the sequence $\mathrm{op}_{1} . \mathrm{op}_{2}$ and more generally the do/undo pair, should have acted as a neutral element for the transposition of $\mathrm{op}_{3}$.

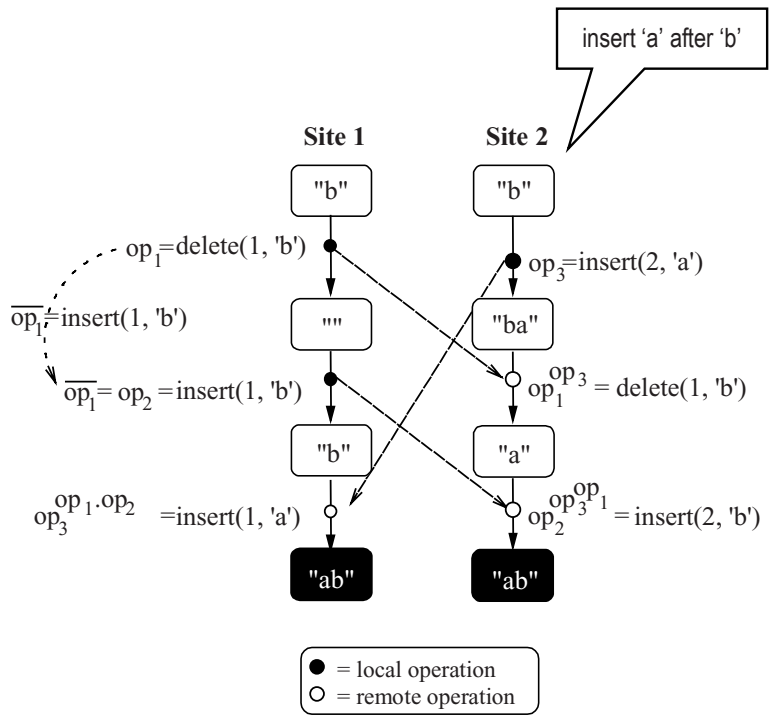

Fig. 6. Situation caused by the failure to respect condition $\mathrm{C} 3$

To ensure that the intention is respected, the forward transposition with an undo operation must verify the general condition $\mathrm{C} 3$.

Condition C3. Neutrality of do/undo pair for the transposition.

Let seq $=o p_{i} \cdot o p_{i+1} \ldots o p_{j-1} \cdot o p_{j}$ and seq $^{\prime}=o p_{i+1}{ }^{\prime} \ldots o p_{j-1}$ be two sequences such that:

- $\forall \mathrm{k} \in[\mathrm{i}+1 . . \mathrm{j}-1], \mathrm{op}_{\mathrm{k}}$ and $\mathrm{op}_{\mathrm{k}}{ }^{\prime}$ achieve the same intention $\mathrm{I}_{\mathrm{k}}$,

- $\mathrm{op}_{\mathrm{j}}$ is the operation which undoes $\mathrm{op}_{\mathrm{i}}$,

then, the forward transposition verifies $\mathrm{C} 3$, iff :

$\forall \mathrm{op}_{\mathrm{k}}, \quad \mathrm{op}_{\mathrm{k}} \mathrm{seq}^{\mathrm{s}} \mathrm{op}_{\mathrm{k}} \mathrm{seq}^{\prime}$

\subsection{Forward Transposition of the Inverse of an Operation (Condition C4)}

When an operation is undone by using the inverse operation, the forward transposition of this inverse operation must verify the condition $\mathrm{C} 4$ which is illustrated by Figure 7. 


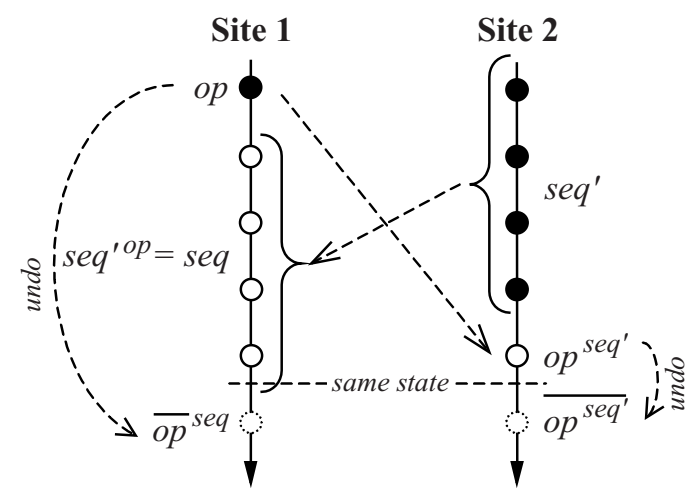

Fig. 7. Illustration of condition $\mathrm{C} 4$

The operation op, local to site 1 , is considered as concurrent to the sequence of operations seq', which is local to site 2 . It is assumed that the sequence seq' was already received on site 1 when the decision is made to undo op. The forward transposition of seq' with op is the sequence seq. On site 2, when op is received, it is forward transposed with seq' to give opseq'. As the executions op.seq on site 1 and seq'.opseq' on site 2 lead to the same state, the operation which undoes op on site 1 must be identical to the one that undoes op on site 2 . In other words, op seq $=\overline{o p}^{\text {seq }}$ must hold.

Condition C4. Forward transposition of the inverse of an operation.

Let op be an operation and seq and seq' two sequences such that:

- Transpose_forward (op, seq') = seq, then the forward transposition verifies $\mathrm{C} 4$, iff:

$$
\text { opseq }=\overline{o p}^{\text {seq }}{ }^{\prime} \text {. }
$$

\subsection{Critical Cases Analysis}

Examples of critical situations were presented in $[2,14,20]$. In these examples, undo is problematic insofar as the use of the naïve algorithm based on Strategy 1 leads to an incorrect result. In fact, as we show in [23], it appears that conditions C3 and/or C4 are not respected.

Therefore, these conditions are necessary to preserve the intentions of all operations. They are also sufficient to preserve intention since $\mathrm{C} 4$ ensures that the intention of undo operations is respected while C3 ensures that the intention of any operation is respected in presence of undo operations. 


\section{Undo Algorithm}

\subsection{Principle}

As previously seen with the undo algorithm based on Strategy 1, the fact of using the inverse operation and forward transposing it with the operations that follow, makes it necessary to verify condition $\mathrm{C} 4$. In practice, it may be very difficult to verify $\mathrm{C} 4$ because an unspecified number of operations is involved. The method that we propose ensures that conditions $\mathrm{C} 3$ and $\mathrm{C} 4$ are automatically verified. In order to achieve this, an undo operation has to be distinguished from a regular operation. We thus introduce the operation undo(op) which expresses the intention to undo operation op. More accurately, a regular operation is specified by its name op, whereas an undo operation is specified by the name undo( ) along with the name of the operation to be undone. Using the notations established in the definition of undo problems, we have:

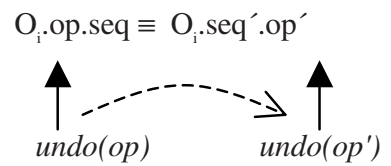

with Transpose_backward $(\mathrm{op}, \mathrm{seq})=\left(\mathrm{seq}^{\prime}, \mathrm{op} \mathrm{p}^{\prime}\right)$,

and thus $\quad \mathrm{op}^{\prime}=\mathrm{op}^{\text {seq' }}$

So, generating the operation undo(op), defined on the $\mathrm{O}_{\mathrm{i}} . \mathrm{op}$ state, and forward transposing it with seq, must be equivalent to generating operation undo( $\left.o p^{\prime}\right)$ defined for the current state $\mathrm{O}_{\mathrm{i}}$.seq'.op' where op' is the last operation executed and achieves the same intention as op. In our method, this is obtained thanks to the definition of forward transposition functions specific to undo, which are such that, $\forall$ op and $\forall$ seq :

$$
\text { Transpose_forward }(\text { seq, undo(op) })=\text { undo }\left(o^{\prime}\right. \text { ') }
$$

The execution of $u n d o\left(o p^{\prime}\right)$ will then consist of executing the inverse operation op on the current state $\mathrm{O}_{\mathrm{i}}$.op.seq. The use of the specific operation undo(op) and the definition of specific transposition functions ensure that $\mathrm{C} 4$ is automatically verified by construction because:

$$
\text { as } \mathrm{op}^{\prime}=\mathrm{op}^{\text {seq', }} \text {, the inverse operations actually verify }
$$

$$
\overline{\mathrm{op}}=\overline{\mathrm{op}^{\mathrm{seq}}}=\mathrm{op}^{\mathrm{seq}} \text {. }
$$

In practice, the operation undo(op') is obtained by successively forward transposing $u n d o(o p)$ with each operation in the sequence seq. The final undo algorithm executed on the site where the decision is made to cancel the operation op is shown in Figure 8-a. Before being appended to the history and broadcast, undo(op') is timestamped with the current state vector of the site as a regular operation $[15,16$, 19].

The other sites which receive the operation undo(op') execute the algorithm shown in Figure 8-b. On these sites, the sequence seqconc of the operations which are concurrent to undo(op') is determined thanks to state vectors associated to each operation (see section 2.1). 


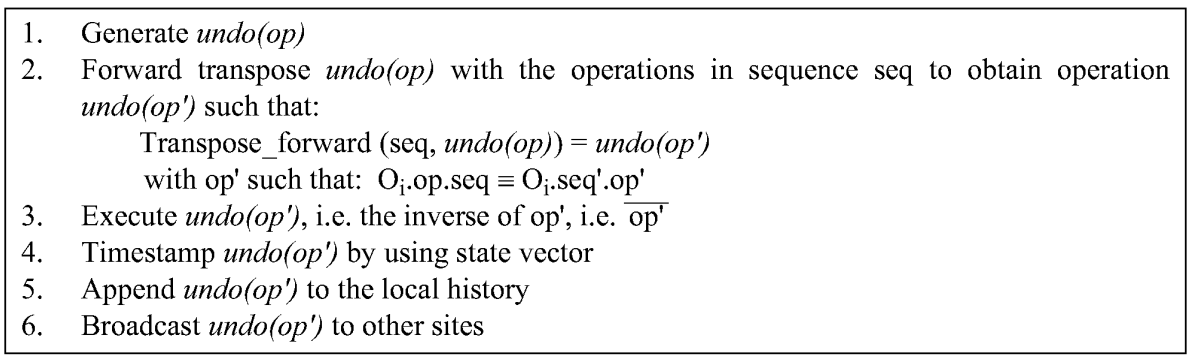

Fig. 8-a. Final Undo Algorithm on the site where the decision is made to cancel op

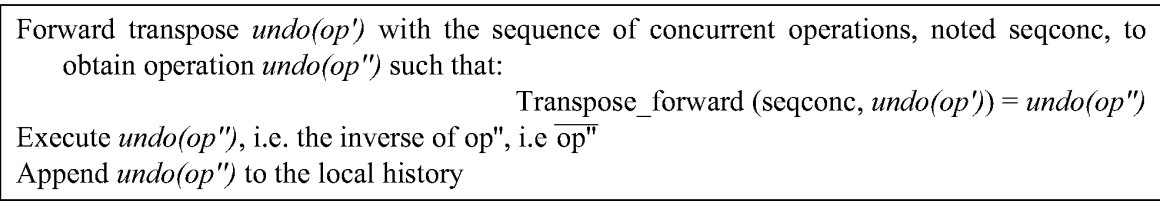

Fig. 8-b. Processing of an undo( $\left.o p^{\prime}\right)$ operation once received by a site

The algorithm ensures that the do/undo pair for the transposition remains neutral. Indeed, taking the equivalence of sequences op.seq and seq'.op' into account, forward transposing an operation $\mathrm{op}_{i}$ with the sequence op.seq.undo(op') amounts to successively forward transposing op $i$ with the operations in sequence seq', then with op' and finally with undo(op'). Achieving this last forward transposition amounts to achieving the inverse of the forward transposition of opi with op', as shown thereafter. The end result is that the forward transposition of opi with the sequence op.seq.undo(op'), which contains the do/undo pair, is reduced to the forward transposition of opi with seq', which ensures that condition C3 is verified. Let us note that the Undo Algorithm works even when the operation op itself is an undo operation. The proof of the Undo Algorithm can be found in [23].

An advantage of our approach lies in the fact that an undo(op') operation, received by a site, can be processed in the same way as a regular operation. According to Figure 3, when OP represents a regular operation op, then $\mathrm{OP}^{\prime}$ is obtained by forward transposing op with the sequence seqconc. Let us imagine that OP represents an undo operation, undo $\left(o p^{\prime}\right)$, then $\mathrm{OP}^{\prime}$ would be obtained by forward transposing undo( $\left.\mathrm{p}^{\prime}\right)$ with seqconc. That exactly matches the processing shown in Figure 8-b. Finally, the processing of a remote operation, whether it is an undo operation or a regular one, received by a site, obeys to the same algorithm.

In a sense, we can say that our method is based on Strategy 2, insofar as operation op' is calculated as if the operation to be undone were the last one to be executed. On another hand, it is also related to Strategy 1 insofar as operation op' is obtained by using forward transposition applied to undo(op) (instead of $\overline{\mathrm{op}}$ as in Strategy 1). The advantage of our approach is that backward transposition is not needed anymore. The only adaptation to be done consists in determining the forward transpositions specific to undo. 


\subsection{Transpositions Specific to Undo}

The method supposes that the forward transpositions written by the programmer are completed to take undo into account. In other words, the Transpose_forward $\left(\mathrm{op}_{1}\right.$, $\mathrm{op}_{2}$ ) function must be specified for the cases where either $\mathrm{op}_{1} \mathrm{or}_{\mathrm{op}}$ (or both) are undo operations. This section shows how the forward transposition can be written in generic form, taking undo into account; this generic form specific to undo does not require any work on behalf of the programmer because it only uses the operations to be undone, their forward transposition and the corresponding inverse operations which have already been defined.

Forward Transposition with an Undo Operation. This section specifies Transpose_forward $\left(\mathrm{op}_{1}, \mathrm{op}_{2}\right)$ when $\mathrm{op}_{1}=$ undo $\left(\mathrm{op}_{3}\right)$. In this case $\mathrm{op}_{2}$ and undo $\left(\mathrm{op}_{3}\right)$ are both defined for the same state (see Figure 9-a). Thus, one can consider that the operation to be undone $\mathrm{op}_{3}$, was executed right before undo( $\left.\mathrm{op}_{3}\right)$ (see Figure 9-b).

a)

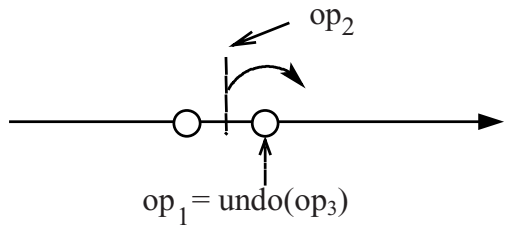

b)

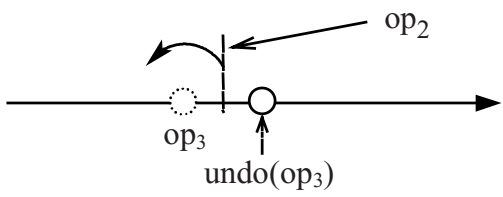

c)

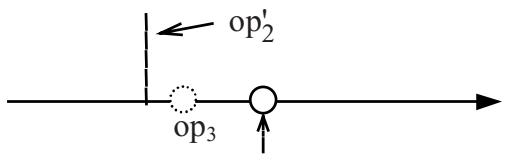

undo $\left(\mathrm{op}_{3}\right)$

d)

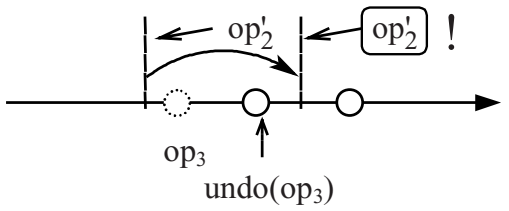

Fig. 9. Forward transposition with an undo operation

As undo $\left(\mathrm{op}_{3}\right)$ amounts to undoing the effect of $\mathrm{op}_{3}$, forward transposing $\mathrm{op}_{2}$ with undo $\left(\mathrm{op}_{3}\right)$ amounts to undoing the effect due to the forward transposition of the operation $\mathrm{op}_{2}{ }^{\prime}$ (to be determined) with $\mathrm{op}_{3}$. For this we need the function which delivers $\mathrm{op}_{2}{ }^{\prime}$ such that Transpose_forward $\left(\mathrm{op}_{3}, \mathrm{op}_{2}{ }^{\prime}\right)=\mathrm{op}_{2}$, where $\mathrm{op}_{2}$ and $\mathrm{op}_{3}$ are known. This function is the inverse of the forward transposition. It is written as Transpose_forward ${ }^{-1}$ and formally defined by:

Transpose_forward ${ }^{-1}\left(\mathrm{op}_{\mathrm{i}}\right.$, Transpose_forward $\left.\left(\mathrm{op}_{\mathrm{i}}, \mathrm{op}_{\mathrm{j}}\right)\right)=\mathrm{op}_{\mathrm{j}}$. 
By applying this function to operations $\mathrm{op}_{3}$ and $\mathrm{op}_{2}$ (see Figure 9-b|c) operation $\mathrm{op}_{2}{ }^{\prime}$ can be obtained by: Transpose_forward-1 $\left(\mathrm{op}_{3}, \mathrm{op}_{2}\right)=\mathrm{op}_{2}{ }^{\prime}$. From its definition, operation $\mathrm{op}_{2}{ }^{\prime}$ achieves the same intention as $\mathrm{op}_{2}$ but is defined for the same state as $\mathrm{op}_{3}$. According to the condition $\mathrm{C} 3$, as the forward transposition of an operation with the pair op/undo(op) must not modify this operation, the forward transposition of $\mathrm{op}_{2}{ }^{\prime}$ with the pair op 3 /undo $\left(\mathrm{op}_{3}\right)$ is quite simply op ${ }_{2}^{\prime}$ (see Figure 9-d). To summarize:

\section{Transpose_forward $\left(\right.$ undo $\left.\left(\mathrm{op}_{3}\right), \mathrm{op}_{2}\right)=$ \\ Transpose_forward ${ }^{-1}\left(\mathrm{op}_{3}, \mathrm{op}_{2}\right)$}

Forward Transposition of an Undo Operation. The specification of Transpose_forward $\left(\mathrm{op}_{1}, \mathrm{op}_{2}\right)$ in the case where $\mathrm{op}_{2}=$ undo $\left(\mathrm{op}_{3}\right)$ proceeds from the same method. It supposes that undo $\left(\mathrm{op}_{3}\right)$ and $\mathrm{op}_{1}$ are defined for the same state, i.e. the state produced by operation $\mathrm{op}_{3}$ (see Figure 10-a).

a)

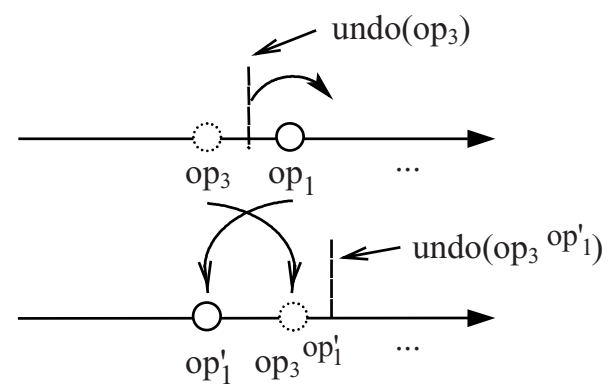

Fig. 10. Forward transposition of an undo operation

To obtain the operation that undoes the effects of $\mathrm{op}_{3}$ after $\mathrm{op}_{1}$ was executed, it suffices to backward transpose the pair $\left(\mathrm{op}_{3}, \mathrm{op}_{1}\right)$ :

$$
\begin{gathered}
\text { Transpose_backward }\left(\mathrm{op}_{3}, \mathrm{op}_{1}\right)=\left(\mathrm{op}_{1}{ }^{\prime}, \mathrm{op}_{3} \mathrm{op}_{1}{ }^{\prime}\right), \\
\text { with } \mathrm{op}_{1}{ }^{\prime}=\text { Tranpose_forward } \\
-1
\end{gathered}
$$

The transposed operation $\mathrm{op}_{3} \mathrm{op}_{1}{ }^{\prime}$ achieves the same intention as $\mathrm{op}_{3}$ if it had been executed just after op $\mathrm{p}_{1}$ (see Figure 10-b). Therefore, the operation we need in order to undo the effect of $\mathrm{op}_{3}$ is undo( $\left.\mathrm{op}_{3} \mathrm{op}_{1}{ }^{\prime}\right)$. To summarize:

$$
\begin{aligned}
& \text { Transpose_forward }\left(\mathrm{op}_{1}, \operatorname{undo}\left(\mathrm{op}_{3}\right)\right)=\operatorname{undo}(\text { Transpose_forward } \\
& \left.\left(\text { Transpose_forward }-1\left(\mathrm{op}_{3}, \mathrm{op}_{1}\right), \mathrm{op}_{3}\right)\right)
\end{aligned}
$$

The complete generic function of the forward transposition which takes undo into account is given in Figure 11. It uses the inverse of the forward transposition. The following specifies how to obtain it when one of the operations in the couple is also an undo operation. 


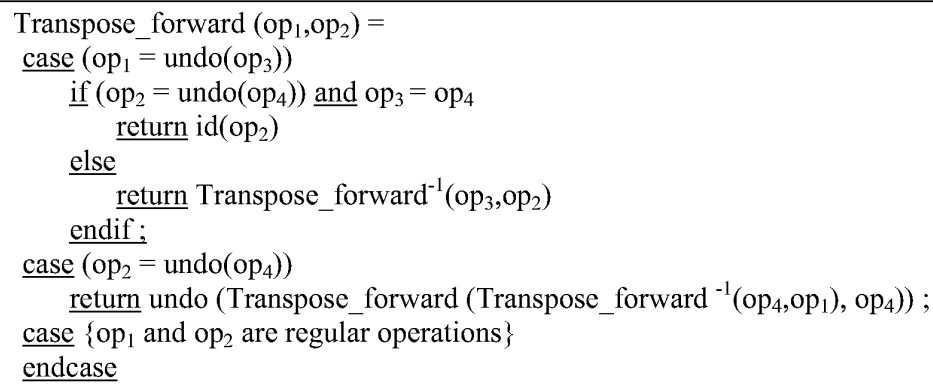

Fig. 11. Forward transposition adapted to undo

Inverse of the Forward Transposition with an Undo Operation. When op 1 is an undo operation, the inverse of the forward transposition, i.e. Transpose_forward $^{-1}\left(\mathrm{op}_{1}, \mathrm{op}_{2}\right)$ can be obtained by the same logic [23]. It is written:

Transpose_forward-1(undo (op), $\left.\mathrm{op}_{2}\right)=\mathrm{op}_{2} \mathrm{op}$

Inverse of the Forward Transposition of an Undo Operation. The inverse of the forward transposition, Transpose_forward-1 $\left(\mathrm{op}_{1}, \mathrm{op}_{2}\right)$, when $\mathrm{op}_{2}$ is an undo operation is difficult to obtain. Given that we know $\mathrm{op}_{1}$ and $\mathrm{op}_{2}$, this amounts to finding $\mathrm{op}_{2}$ such that Transpose_forward $\left(\mathrm{op}_{1}, \mathrm{op}_{2}{ }^{\prime}\right)=\mathrm{op}_{2}$. As $\mathrm{op}_{2}$ is an undo operation, written as undo $\left(\mathrm{op}_{3}\right)$, then $\mathrm{op}_{2}{ }^{\prime}$ is also an undo operation, written as undo(op $\left.{ }_{3}{ }^{\prime}\right)$. Thus, finding $\mathrm{op}_{2}{ }^{\prime}$ amounts to finding $\mathrm{op}_{3}{ }^{\prime}$. When considering the relation previously established to calculate the forward transposition of an undo operation, operation $\mathrm{op}_{2}$ is given by the following:

$\mathrm{op}_{2}=$ undo $\left(\right.$ Transpose_forward $\left(\right.$ Transpose_forward $\left.\left.{ }^{-1}\left(\mathrm{op}_{3}{ }^{\prime}, \mathrm{op}_{1}\right), \mathrm{op}_{3}{ }^{\prime}\right)\right)$.

In addition, when considering $\mathrm{op}_{2}=$ undo $\left(\mathrm{op}_{3}\right)$, given that we know $\mathrm{op}_{2}$, so we know $\mathrm{op}_{3}$. As $\mathrm{op}_{3}$ is given by $\mathrm{op}_{3}=$ Transpose_forward (Transpose_forward ${ }^{-1}$ (op3', $\mathrm{op}_{1}$ ), $\left.\mathrm{op}_{3}{ }^{\prime}\right)$, finding $\mathrm{op}_{3}{ }^{\prime}$ would be necessary before knowing Transpose_forward$1\left(\mathrm{op}_{3}{ }^{\prime}, \mathrm{op}_{1}\right)$. This evaluation is impossible using operation op $\mathrm{p}_{1}$ alone. In fact, in order to obtain the result, we need to refer to the history of the site [23] and to reorder the operations to obtain an equivalent history containing the operation $\mathrm{op}_{2}{ }^{\prime}$, i.e. undo $\left(\mathrm{op}_{3}{ }^{\prime}\right)$.

\section{Illustration of the Undo Algorithm}

In this section we show on an example how our Undo Algorithm works. The example, referred as Insert-Insert-Tie Dilemma [20], corresponds to a situation illustrated in Figure 12-a. User 1 on site 1 deletes the character ' $b$ ', while user 2 on site 2 inserts 'a' 

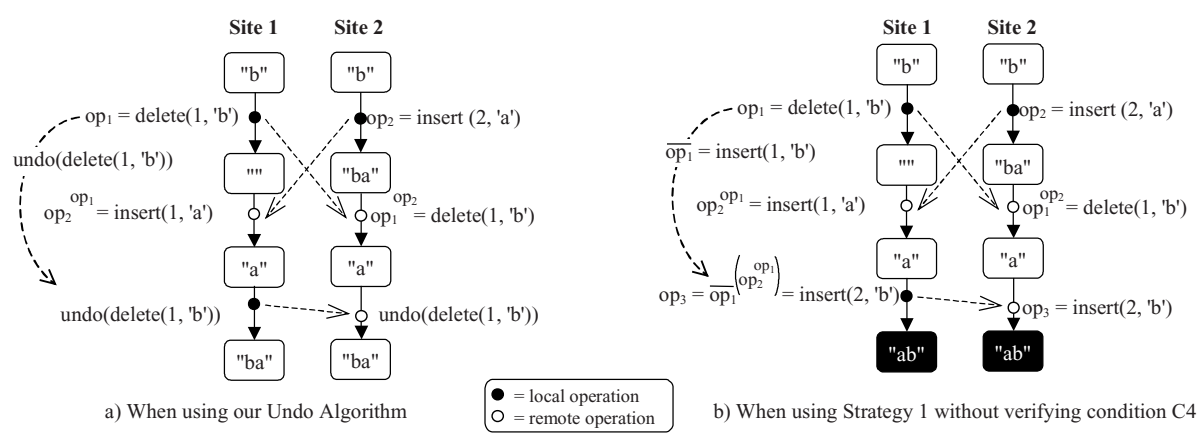

b) When using Strategy 1 without verifying condition C4

Fig. 12. Insert-Insert-Tie example

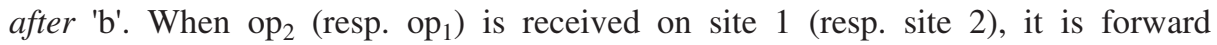
transposed with op $_{1}$ (resp. op $_{2}$ ) before being executed. Respecting condition $\mathrm{C} 1$ ensures that the copies converge towards the same state "a". Let us suppose that user 1 then decides to cancel operation $\mathrm{op}_{1}$. The application of our Undo Algorithm (see Figure 8-a), with operation op corresponding to delete $\left(1, b^{\prime}\right)$, gives the following statements:

Step 1. Generate undo(delete $\left.\left(1, b^{\prime}\right)\right)$.

Step 2. Seeing that seq = insert $(1$, 'a'), thus compute:

Transpose_forward (insert(1, 'a'), undo(delete $\left.\left(1, b^{\prime}\right)\right)$ ).

Referring to Figure 11, insert(1, 'a') corresponds to op ${ }_{1}$ and $\operatorname{undo}\left(\operatorname{delete}\left(1,{ }^{\prime}{ }^{\prime}\right)\right)$ to $\mathrm{op}_{2}$, which leads to the case where $\mathrm{op}_{2}=\operatorname{undo}\left(\mathrm{op}_{4}\right)$, with $\mathrm{op}_{4}=\operatorname{delete}\left(1, \mathrm{~b}^{\prime}\right)$. According to these notations, the result to be computed is:

undo (Transpose_forward(Transpose_forward $\left.{ }^{-1}\left(\mathrm{op}_{4} o \mathrm{p}_{1}\right), \mathrm{op}_{4}\right)$ )

which needs to compute:

i) the inverse of Transpose_forward(delete(1, 'b'), insert(1, 'a')); the result is insert(2, 'a');

ii) Transpose_forward (insert(2, 'a'), delete(1, 'b')); the result is delete(1, 'b').

The final result of step 2 is undo(delete $\left.\left(1, b^{\prime}\right)\right)$.

Step 3. Execute the inverse of delete(1, 'b'), that is insert(1, 'b'), which leads to the final state "ba".

After being timestamped and appended to the history, the resulting operation of step 2 , undo(delete $\left.\left(1, b^{\prime}\right)\right)$, is broadcast to site 2 . When it is received on this site, the algorithm shown in Figure 8-b is applied. As there is no concurrent operation, the inverse of delete $\left(1, b^{\prime}\right)$, that is insert( $\left.1, b^{\prime}\right)$, is directly executed and leads to the same state "ba".

The application of the naïve algorithm based on Strategy 1 (see Figure 4) and illustrated in Figure 12-b would lead to a wrong result. The operation op $1=\operatorname{insert}(1$, 'b') would be generated then forward transposed with $\mathrm{op}_{2} \mathrm{op}_{1}$ to obtain $\mathrm{op}_{3}=$ $\mathrm{op}_{1} \mathrm{op}_{2} \mathrm{op}_{1}=\operatorname{insert}\left(2, \mathrm{~b}^{\prime}\right)$ whose execution would lead to state "ab". Operation $\mathrm{op}_{3}$, broadcast to site 2 would be executed as it is on this site. Although the copies would converge towards the same state, the undoing of op 1 would lead to an incorrect state since the intention of user 2 , namely 'a' placed after ' $b$ ', would not be respected in the 
final state. The reason for this anomaly is due to the failure to respect condition $\mathrm{C} 4$. The operation executed on site 2 to undo operation $\mathrm{op}_{1} \mathrm{op}_{2}=\operatorname{delete}(1, \mathrm{~b}$ '), should be identical to the inverse operation op $1 \mathrm{OP}_{2}=\operatorname{insert}\left(1, \mathrm{~b}^{\prime}\right)$, which is not the case here.

It results that, when using our Undo Algorithm, the example referred as Insert-Insert-Tie Dilemma [20], is not a critical situation anymore.

\section{Comparison with Existing Approaches}

The adOPTed algorithm [13] is based on the use of Operational Transformations and on a multidimensional history associated with each copy. This history is represented by a graph where each dimension relates to the operations generated by a given user. An extension to the adOPTed algorithm, based on the naïve algorithm, is proposed in [14] to enable a user to undo operations. However, the extension restricts undo to local operations only on condition that they are undone according to the inverse chronological order. As a result of these limitations an operation op that undoes op can only be separated from the operation op in a given dimension by a sequence containing do/undo pairs only. This characteristic facilitates the adaptation of the forward transposition function (called translateRequest) so that it can take the do/ undo pairs into account and ensure that condition C3 is verified. Moreover, thanks to the multidimensional history, for any operation op concurrent to a sequence seq, the operation opseq is directly available. Therefore, the verification of condition $\mathrm{C} 4$ is unnecessary because the undoing of op is achieved by generating and executing opseq. However, the undoing of local operations according to their inverse chronological order remains a restrictive solution.

The DistEdit Selective Undo algorithm [12] implements Strategy 2 and only ensures that a condition equivalent to $\mathrm{C} 3$ is met, since condition $\mathrm{C} 4$ is automatically satisfied by this Strategy.

The REDUCE system [19] is based on Operational Transformations and on a linear history associated with each copy of the object. The principle of the undo algorithm, called ANYUNDO [20, 21], is a naïve algorithm adaptation obtained by grouping an operation and the corresponding undo in the history. This adaptation makes it possible to ensure the neutrality of the do/undo pairs during the transposition of an operation and, therefore, to ensure that condition C3 is met. More precisely, undoing op is achieved by: generating op, transposing it forward with the sequence seq of the operations executed after op; and executing and broadcasting the operation obtained $\mathrm{op}^{\mathrm{seq}}$. Grouping op and the corresponding undo operation to obtain the do/undo pair, written as op ${ }^{*}$, is achieved by backward transposing the pair (seq, opseq). In [20], the lack of a timestamp for an undo operation makes it impossible to distinguish between concurrent operations and causally dependent operations; that may result in violating user intention and lead to the divergence of the copies. In [21], this mistake is corrected and conditions IP1, IP2 and IP3 equivalent to conditions C3 and C4 are retrieved. However, the correction is obtained by extending the ANYUNDO algorithm with undo specific additive treatments. The interest of our approach lies in the generality of the processing of a remote operation whether it is an undo operation or a regular one. This generality is obtained thanks to the introduction of a specific undo operation which obeys to the same processing as a regular operation. 


\section{Conclusion}

This article reviews the problems arising from the cancellation of an operation in distributed collaborative environments that use Operational Transformations. Traditionally, undo operations were often limited to the handling of the inverse operation. However, we show that when concurrency occurs, this approach is insufficient to ensure that the copies converge and it fails to respect user intention. Moreover, the forward transposition function must verify two conditions, which we have highlighted. However, these conditions are difficult to check in practice. In this context, we proposed a general undo algorithm for which these conditions are automatically met. Its originality lies in its capacity to consider undo as a specific operation that requires the adaptation of the Operational Transformations, an adaptation for which we give a generic specification. This algorithm makes it possible to undo any operation, local or remote, in all situations of concurrency, including those that are widely considered as problems. The paper concludes with a comparison with existing algorithms.

\section{References}

[1] Abowd G. D. , Dix A. J.; "Giving Undo Attention"; Interacting with Computers, vol. 4, n 3, pp. 317-342, 1992.

[2] Berlage T.; "A Selective Undo Mechanism for Graphical User Interfaces Based on Command Objects"; ACM Transactions on Computer-Human Interaction, vol. 1, n 3, pp. 269-294, 1994.

[3] Choudhary R., Dewan P.; "A general Multi-User Undo/Redo Model"; Proc. of European Conference on Computer Supported Work (ECSCW'95), Stockholm, October 1995, pp. 231-246.

[4] Ellis C.A., Gibbs S.J.; "Concurency Control in Groupware Systems"; Proc. ACM Int. Conf. on Management of Data (SIGMOD'89), Seattle, May 1989, pp.399-407.

[5] Ellis C.A., Gibbs S.J., Rein G.L.; "Groupware: Some issues and experiences"; Commun. ACM, January 1991, vol.34, n 1, pp.39-59.

[6] Ignat C., Norrie M.C.; "Customizable Collaborative Editor Relying on the treeOPT Algorithm"; Proc. $8^{\text {th }}$ European Conf. on Computer Supported Cooperative Work (ECSCW'03), Helsinki, September 2003.

[7] Li D., Zhou L., Muntz R. R.; "A new paradigm of user intention preservation in realtime collaborative editing systems"; Proc. $7^{\text {th }}$ Int. Conf. on Parallel and Distributed Systems (PADS'00), Iwate, Japan, July 2000.

[8] Mancini R., Dix A., Levialdi S.; "Reflections on Undo"; Technical report, University of Hudders eld, 1996.

[9] Molli P., Skaf-Molli H., Oster G.; "Divergence Awarenes for Virtual Team through the Web"; Proc 6th Int. Conf. on Integrated Design and Process Technology (IDPT'02), June 2002

[10] Palmer C.R., Cormack G.V.; "Operation Transforms for a Distributed Shared Spreadsheet"; Proc. ACM Int. Conf. on Computer Supported Cooperative Work (CSCW'98), Seattle, November 1998, pp. 69-78, 1998.

[11] Prakash A., Knister M.J.; "Undoing Actions in Collaborative Work"; Proc. ACM Int. Conf. on Computer Supported Cooperative Work (CSCW'92), November 1992, pp. 273280.

[12] Prakash A., Knister M.J.; "A Framework for Undoing Actions in Collaborative Systems"; ACM Transactions on Computer-Human Interaction, vol. 1, n 4, pp. 295-330, 1994. 
[13] Ressel M., Nitssche-Ruhland D., Gunzenhäuser R.; "An Integrating, Transformationoriented Approach to Concurrency Control and Undo in Group Editors"; Proc. ACM Int. Conf. on Computer Supported Cooperative Work (CSCW'96), Boston, November 1996, pp. 288-297.

[14] Ressel M., Gunzenhäuser R.; "Reducing the Problems of Group Undo"; Proc. ACM Int. Conf. on Supporting Group Work (GROUP'99), Phoenix, November 1999, pp.131-139.

[15] Suleiman M., Cart M., Ferrié J.; "Serialization of Concurrent Operations in a Distributed Collaborative Environment"; Proc. ACM Int. Conf. on Supporting Group Work (GROUP'97), Phoenix, November 1997, pp. 435-445.

[16] Suleiman M., Cart M., Ferrié J.; "Concurrent Operations in a Distributed and Mobile Collaborative Environment"; Proc. 14th IEEE Int. Conf. on Data Engineering (IEEE / ICDE'98), Orlando, February 1998, pp. 36-45.

[17] Suleiman M.; "Sérialisation des opérations concurrentes dans les systèmes collaboratifs répartis"; Doctoral thesis, Université de Montpellier 2, July 1998.

[18] Sun C., Jia X., Yang Y., Zhang Y.; "A generic operation transformation schema for consistency maintenance in real-time cooperative editing systems"; Proc. ACM Int. Conf. on Supporting Group Work (GROUP'97), Phoenix, November 1997, pp.425-434.

[19] Sun C., Ellis C.S.; "Operational Transformation in Real-Time Group Editors : Issues, Algorithms and Achievements"; Proc. ACM Int. Conf. on Computer Supported Cooperative Work (CSCW'98), Seattle, November 1998, pp. 59-68.

[20] Sun C.; "Undo Any Operation at Any Time in Group Editors "; Proc. ACM Conf. on Computer Supported Cooperative Work (CSCW'00), Philadelphia, Pennsylvania, December 2-6, 2000, pp. 191-200.

[21] Sun C.; "Undo as Concurrent Inverse in Group Editors"; ACM Transactions on Computer-Human Interaction, vol. 9, n 4, pp. 309-361, December 2002.

[22] Vidot N., Cart M., Ferrié J., Suleiman M.; "Copies convergence in a distributed real-time collaborative environment"; Proc. ACM Int. Conf. on Computer Supported Cooperative Work (CSCW'00), Philadelphia, Pennsylvania, December 2-6, 2000, pp. 171-180.

[23] Vidot N.; "Convergence des Copies dans les Environnements Collaboratifs Répartis"; Doctoral thesis, Université de Montpellier 2, September 2002. 